\title{
Reddening map of the Large Magellanic Cloud bar region ${ }^{\star}$
}

\begin{abstract}
A. Subramaniam
Indian Institute of Astrophysics, II Block, Koramangala, Bangalore 560034, India

e-mail: purni@iiap.res.in

Received 12 May 2004 / Accepted 16 September 2004

Abstract. $E(V-I)$ reddening values for 1123 locations in the bar region of the Large Magellanic Cloud are presented. $V$, I photometry of red clump stars identified in the Optical Gravitational Lensing Experiment II catalogue of LMC were used to estimate reddening. $E(V-I)$ values were estimated as the difference between observed and the characteristic values of $(V-I)$ for the red clump population in a given region. It is found that most of the regions in the bar have reddening values less than $0.1 \mathrm{mag}$ with only a few locations having values more than $0.2 \mathrm{mag}$. The eastern side of the bar is found to be more reddened when compared to the western side, with similar and relatively small values for differential reddening as in most parts of the bar. Increased reddening in the eastern end of the bar could be caused by a small fraction of the H I clouds in the line of sight. A high density of HI clouds located in the eastern end of the bar should have caused very high reddening in these regions, whereas only a relatively small increase in the reddening is estimated. This indicates that most of the $\mathrm{H}$ I clouds in this direction are likely to be located behind the bar.
\end{abstract}

Key words. galaxies: Magellanic Clouds - dust, extinction

\section{Introduction}

The Large Magellanic Cloud (LMC) is the nearest galaxy where one can resolve individual stars and study them. Since the metallicity of the LMC is about one third of the metallicity of our Galaxy, the dependence of various processes on metallicity can be understood by studying stars in various evolutionary phases in the LMC. One of the main hurdles in deriving the observed characteristics is in estimating reddening in the line of sight towards them. Properties of similar stars located in different parts of the LMC can be compared only if a reliable estimation of reddening is available for various locations. Thus a map of reddening with sufficient spatial resolution is necessary. In recent years, the LMC has been very thoroughly studied using various surveys, for example, the Optical Gravitational Lensing Experiment II (OGLE II Udalski et al. 2000), Magellanic Clouds Photometric Survey (MCPS, Zaritsky et al. 1997), Massive Compact Halo Objects (MACHO) survey (Alcock et al. 2000). These surveys can be used to map reddening in the LMC. Since data from a survey catalogue are homogeneous, systematic errors in the reddening estimation from such a catalogue will be minimal.

Some of the previous efforts to estimate reddening using survey catalogues are listed below. Sumi (2004) estimated $E(V-I)$ reddening and extinction map towards the Galactic bulge using the OGLE II data towards the bulge. Popowski et al. (2003) presented a reddening map of the

* Full Table 1 is only available in electronic form at the CDS via anonymous ftp to cdsarc.u-strasbg.fr $(130.79 .128 .5)$ or via http://cdsweb.u-strasbg.fr/cgi-bin/qcat?/J/A+A/430/421
Galactic bulge/bar based on $(V-R)$ colours from the photometry of the MACHO microlensing survey. Wozniak \& Stanek (1996) used red clump stars in the Galactic bulge from the OGLE II survey to estimate reddening curve and Stanek (1996) used their method to obtain a high resolution reddening map of Baade's window.

A high resolution reddening map of the LMC bar region estimated using the OGLE II catalogue is presented here. In this study, we used red clump stars as a tracer to estimate reddening. Red clump stars in the bar region of the LMC can be assumed to belong to a small range of age and metallicity. Studies by Subramaniam \& Anupama (2002), Olsen \& Salyk (2002) and van der Marel \& Cioni (2001) found no noticeable change in the age or metallicity of the red clump population in the LMC. Thus red clump stars can be considered to be a homogeneous population. This assumption is used to choose a characteristic luminosity and colour for the population. The red clump stars are considered as standard candles and have been widely used for estimation of distance to the LMC (Stanek et al. 1998; Udalski et al. 1998; Cole 1998). In these studies, characteristic luminosity of the population is used to estimate distance. In the present study, their characteristic colour is used to estimate reddening. The estimates of reddening obtained here are average values of red clump stars located in a given region. The relative change of this average value across the bar of the LMC is a good indicator of the variation in reddening across the bar region. There may also be differential reddening within the LMC, for stars located in the same region. The error in the estimation of reddening is a proxy for the amount of differential reddening in that direction. Reddening results of this analysis have been 


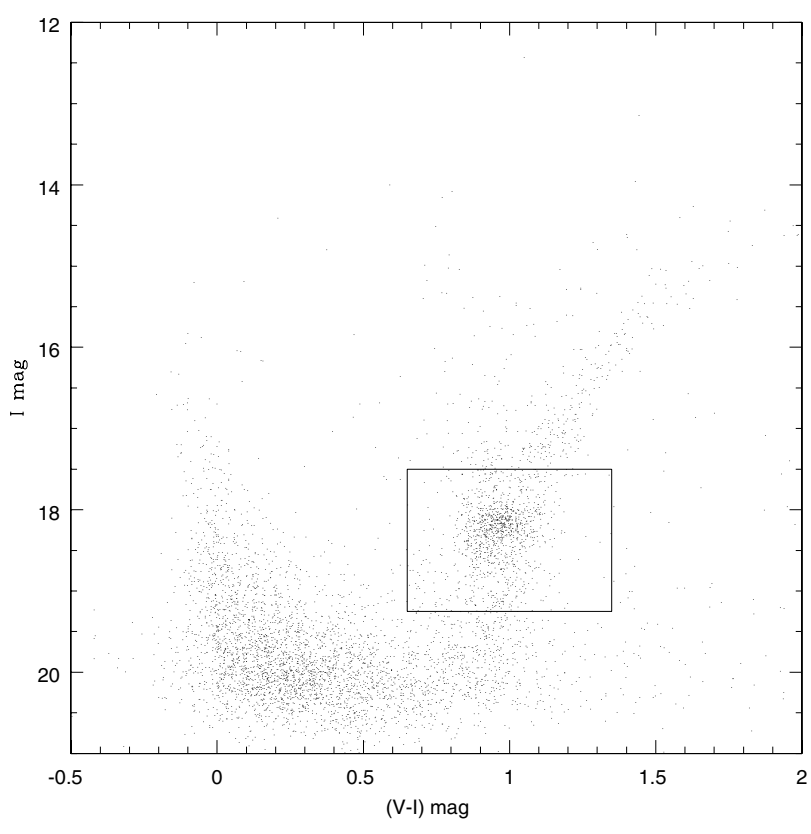

Fig. 1. A typical $I$ (mag) versus $(V-I)(\mathrm{mag}) \mathrm{CMD}$ is shown. The red clump stars are identified as stars within the box shown.

used by Subramaniam (2003) to understand the structure of the bar. Detailed reddening results are presented here so that they could be used for other studies.

\section{Data}

The OGLE II survey (Udalski et al. 2000) consists of photometric data of 7 million stars in $B, V$ and $I$ pass bands in the central 5.7 square degree of the LMC. The catalogue presented data for 26 regions, of which 21 regions were studied here, which are located within 2.5 degree from the optical center of the LMC $\left(\mathrm{RA}=05^{\mathrm{h}} 19^{\mathrm{m}} 38^{\mathrm{s}} .0\right.$, Dec $=-69^{\circ} 27^{\prime} 5^{\prime \prime} .2$, de Vaucouleurs \& Freeman 1973). In this study, the total observed region was divided into 1344 sections. Each strip was divided into $4 \times 16$ square sections, each having an area of $3.56 \times 3.56 \mathrm{arcmin}^{2}$. Thus each of the 21 strips was divided into 64 square sections, giving rise to 1344 sections. For each section, an $I$ versus $(V-I)$ colour magnitude diagram (CMD) was constructed. Red clump stars were identified using $I$ vs. $(V-I) \mathrm{CMD}$, as stars within the box, as shown in Fig. 1. Blue and red boundaries of the box in $(V-I)$ are $0.65 \mathrm{mag}$ and $1.35 \mathrm{mag}$ respectively, whereas the bright and faint boundaries in $I$ mag are 17.5 mag and 19.25 mag respectively. The position of the red clump population was found to be located within this box in almost all the locations and hence the location of this box was kept fixed in the CMD. As the density of stars is not uniform in the bar, the number of red clump stars also varies accordingly. The number of red clump stars identified was found to vary between 800-3000 with an average of 1500 red clump stars per region. The photometric data suffer from incompleteness in the data due to crowding effects, and the incompleteness in the data in $I$ and $V$ pass bands are tabulated in Udalski et al. (2000). Within the box of red clump stars, a two-dimensional grid was formed using a bin size of $0.010 \mathrm{mag}$ in $(V-I)$ and $0.025 \mathrm{mag}$ in $I$ magnitude. For each location in the grid, there would be two values of incompleteness, one from the $V$ frame and the other from the $I$ frame. The lower of the two values was considered as the true incompleteness and applied to obtain the actual number of stars. The selected value of incompleteness varies within the box as well as with the location of the region in the LMC bar. In crowded regions, the minimum value of completeness was found to be $81 \%$, whereas for least crowded regions, the minimum value was $94 \%$. The above values refer to the bottomright corner of the box and also indicate the scaling adopted to obtain the actual value of red clump stars from the observed number in the CMD. Thus the estimated incompleteness factor for each region was applied and the total number of red clump stars was estimated for all the 1344 sections.

\section{Estimation of the reddening map}

It is evident from the CMD that red clump stars have a spread in luminosity and colour within the box in the CMD. The number distribution of stars with respect to $(V-I)$ colour shows a Gaussian-like distribution, with a peak and a width. The peak of the profile can be considered to be due to the major fraction of the red clump stars in the region considered. This peak of the distribution would get shifted towards redder colours due to reddening, with respect to its characteristic colour. Thus by estimating the shift in the peak of the distribution, the average reddening towards the location can be estimated. The spread in profile could be due to factors like differential reddening, presence of binaries (real as well as line of sight) and difference in metallicity. The differential reddening is due to the difference in reddening towards stars located in a region in the same line of sight, because of gas/dust located between the closest and the farthest red clump star in the line of sight. If two stars are located in the same line of sight, which cannot be resolved, then the estimated magnitude and colour are the integrated values of the two stars. If we consider a star in the red clump and another star not in the red clump, then these two stars have different intrinsic $(V-I)$ and $I$ magnitudes. If these two stars happen to be in the same line of sight, then the integrated values of the $I$ magnitude and $(V-I)$ colour will not be the same as that of the red clump star. The resulting change will depend on the location of the second star in the CMD. This is true for real binaries also. The net effect is to produce a scatter around the observed clump in the CMD, increasing the width of the profile.

In order to estimate the number distribution of red clump stars, stars were binned using a bin size of 0.010 mag. The distribution was then fitted with a Gaussian + quadratic function. A similar function was used by Udalski et al. (1999) as well as Olsen \& Salyk (2002) to fit the red clump distribution. One such distribution for a location is shown in Fig. 2. The histogram represents the observed number distribution and the curve represents the best fit function. Estimated values of the peak, its error and goodness of fit are also indicated in the figure. This procedure was repeated for all the $1344 \mathrm{sec}-$ tions. Goodness of fit was estimated using reduced $\chi^{2}$ values and these values were found to be similar for more than $90 \%$ of the regions. Some regions showed a poor fit along with relatively higher values for width of the profile. These regions were 


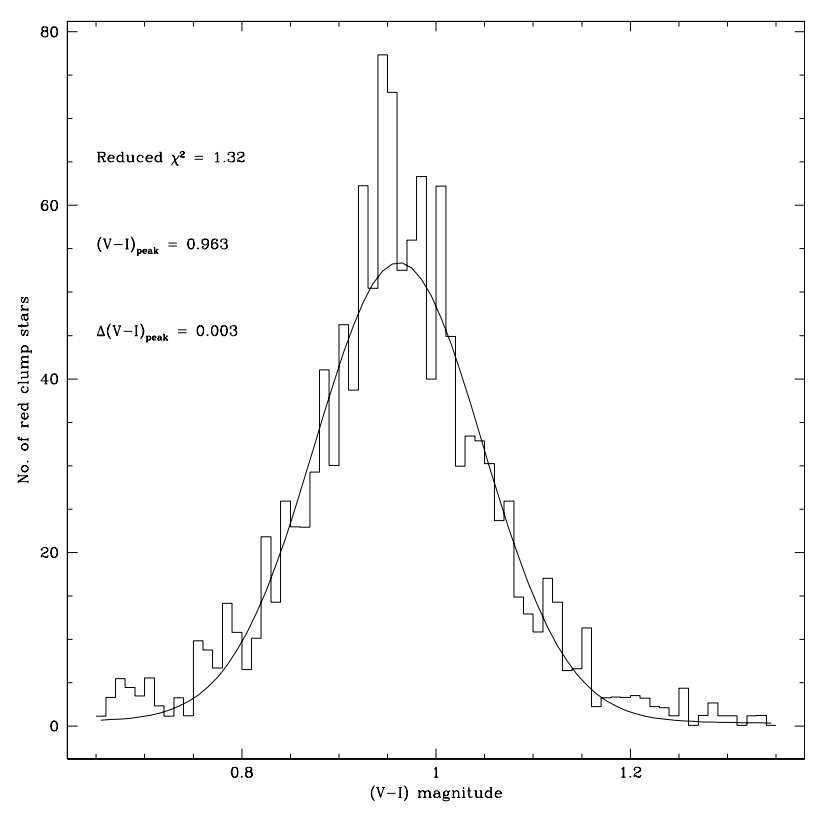

Fig. 2. Number distribution of red clump stars as a function of $(V-I)$ mag is shown as a histogram. The fitted curve is also shown. The estimated values of the peak of the function, error in the estimation of the peak and reduced $\chi^{2}$ value of the fit are also shown in the figure. This data is obtained within a area of $3.56 \times 3.56 \mathrm{arcmin}^{2}$ centered at the location $\mathrm{RA}=5^{\mathrm{h}} 32^{\mathrm{m}} 45^{\mathrm{s}}$ and $\mathrm{Dec}=-70^{\circ} 25^{\prime} 37^{\prime \prime}$.

not included for reddening estimation. The average value of $\chi^{2}$ for the fit was found to be 1.39. Regions with a fit of the distribution worse than 2.5 were rejected and the number of regions for reddening estimation was reduced to 1123 .

The distribution of reduced $\chi^{2}, \sigma$ and error in the estimation of $(V-I)_{\text {peak }}$ are shown as a function of $\alpha$ in Fig. 3. Since the data spans about 12 degree in right ascension, $\alpha$, and the span in declination, $\delta$ is only about 2 degrees, the errors are shown as a function of $\alpha$. Reduced $\chi^{2}$ values were found to be mostly less than 2.0. Slightly higher values were found between $78^{\circ}$ and $82^{\circ}$ and very high values were found eastward of $84^{\circ}$. The values of $\sigma$ as shown in the middle panel indicates that higher values are seen only in the eastern side. The error in $(V-I)_{\text {peak }}$ as shown in the lower panel, does not show any trend between $\alpha=75^{\circ}-84^{\circ}$. Locations eastward of $84^{\circ}$ are found to show higher reddening and the maximum value of error was found to be $0.005 \mathrm{mag}$. A significant number of locations between $\alpha=$ $84^{\circ}-87^{\circ}$ are found to show poor fits. One of the reasons for the poor fit could be that there is a considerable amount of internal reddening within the LMC in these regions. This is supported by the fact that a large amount of $\mathrm{H}$ I gas is found in the radio observations in the eastern end of the bar. Subramaniam (2004) found that between $\alpha=84^{\circ}-85^{\circ}$, the red clump stars have relatively large depth in the line of sight. The above two effects could result in broadening of the peak and an increase in the value of $\sigma$. In general, observed profile could shift considerably from the fitting function, resulting in poor fits.

Peak values of $(V-I)$ colour at each location was used to estimate reddening. Reddening was calculated using the relation $E(V-I)=(V-I)_{\mathrm{obs}}-0.92 \mathrm{mag}$. The intrinsic colour of red clump stars was assumed to be 0.92 mag
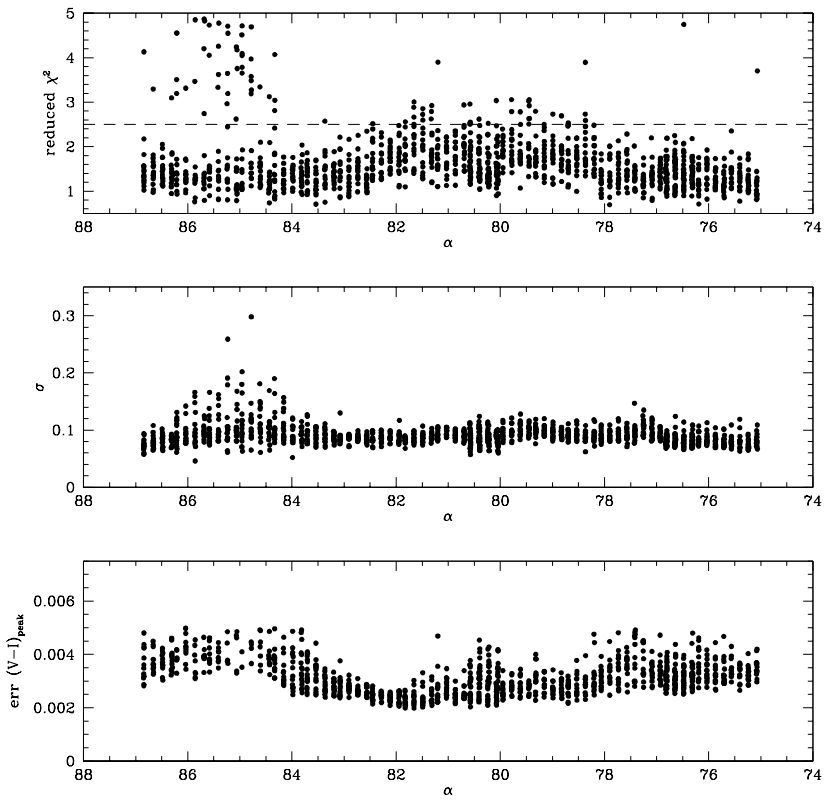

Fig. 3. The estimates of reduced $\chi^{2}$ value, width of the profile $(\sigma)$ and error in the peak value of $(V-I)$ are shown as a function of $\alpha$. In the top panel, the dashed line indicates the cut-off value of reduced $\chi^{2}$ value.

Table 1. A subsample of $E(V-I)$ reddening values. The complete table is available through CDS.

\begin{tabular}{llll}
\hline \hline $\begin{array}{l}\alpha(\mathrm{J} 2000) \\
\text { (degrees) }\end{array}$ & $\begin{array}{l}\delta(\mathrm{J} 2000) \\
\text { (degrees) }\end{array}$ & $\begin{array}{l}E(\mathrm{~V}-I) \\
(\mathrm{mag})\end{array}$ & $\begin{array}{l}\text { Error } \\
(\mathrm{mag})\end{array}$ \\
\hline 5017 & -692034 & 0.084 & 0.004 \\
5017 & -691658 & 0.085 & 0.003 \\
5017 & -69247 & 0.111 & 0.004 \\
5017 & -691333 & 0.058 & 0.003 \\
5018 & -69628 & 0.057 & 0.003 \\
5018 & -69101 & 0.069 & 0.003 \\
5018 & -69252 & 0.095 & 0.004 \\
5019 & -685923 & 0.088 & 0.004 \\
5019 & -685219 & 0.067 & 0.004 \\
5019 & -685548 & 0.087 & 0.004 \\
5019 & -684517 & 0.054 & 0.003 \\
5019 & -684846 & 0.061 & 0.004 \\
5020 & -684145 & 0.068 & 0.004 \\
5020 & -683816 & 0.059 & 0.003 \\
\hline
\end{tabular}

(Olsen \& Salyk 2002). Thus $E(V-I)$ values were estimated for 1123 locations in the LMC and are tabulated in Table 1.

In Fig. 4, locations of 1123 sections for which $E(V-I)$ could be reliably estimated are shown. It can be seen that only $16 \%$ of the total number of regions are left out. In the figure, different colours are used to indicate different ranges in the reddening value. The central regions show low and similar reddening values. Towards the western end, some regions were found to show higher reddening. The eastern end is found to show higher values of reddening, and the highest estimated values of reddening were found for regions located here. The most striking feature of this figure is that higher reddening is found at the ends of the bar and not in the central regions. 


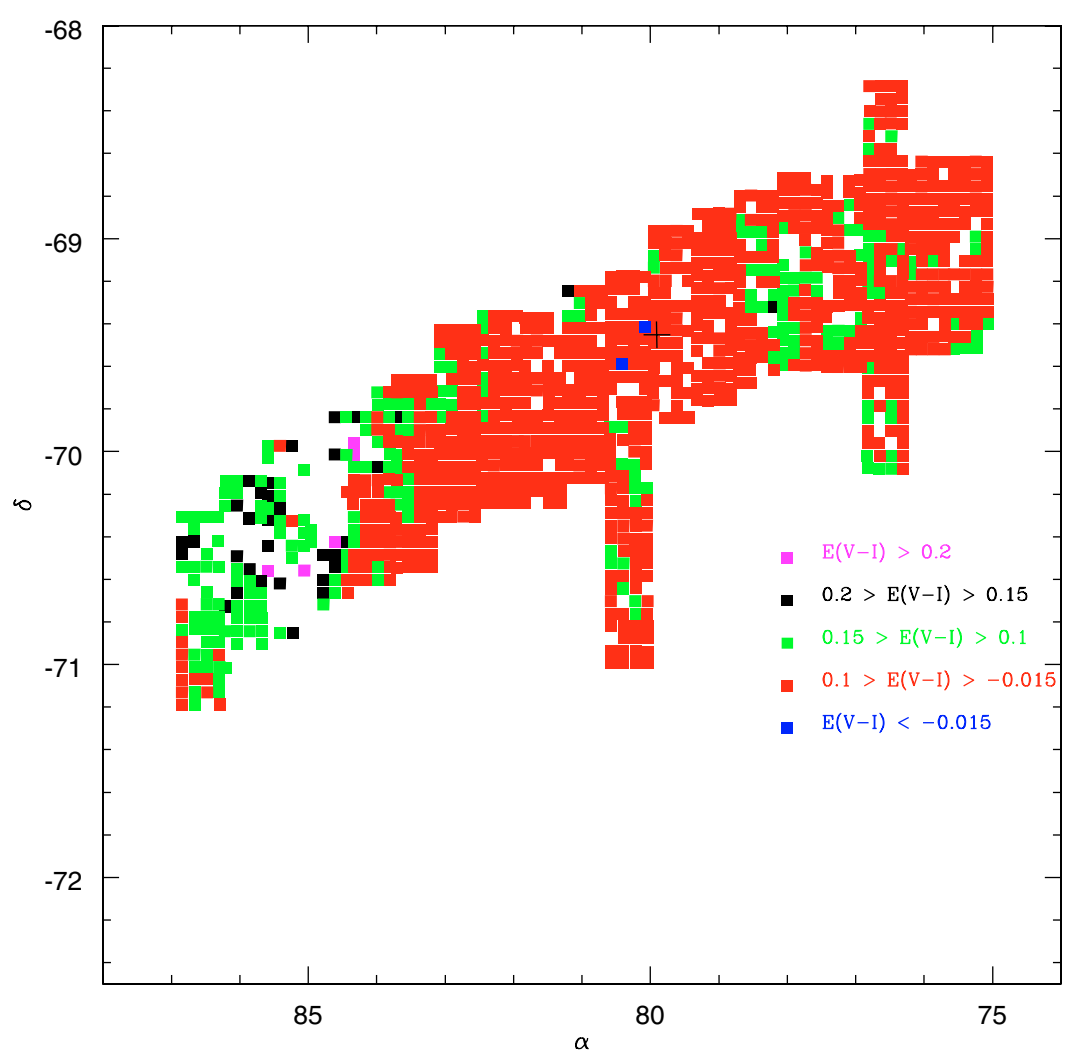

Fig. 4. Location of the sections for which the $E(V-I)$ reddening values were estimated. Different colours are used to denote locations with different values of reddening, which is explained in the figure. The missing points are due to locations for which reliable estimates of reddening could not be obtained. The plus sign denotes the optical center of the LMC.

The total average reddening was also estimated and was found to be $E(V-I)=0.08 \pm 0.04$ mag. This indicates that in general, reddening towards the bar regions is low. Also, the relatively low value of dispersion indicates that the amount of differential reddening across the face of the LMC bar is insignificant.

Reddening values are plotted as a function of $\alpha$ and $\delta$ in Fig. 5. It can be readily seen that, on an average, the $E(V-I)$ value is $\leq 0.1 \mathrm{mag}$ for most of the regions. The eastern regions have slightly higher values of reddening up to $0.2 \mathrm{mag}$. Only a few regions were found to have $E(V-I)$ values higher than $0.2 \mathrm{mag}$. It can be seen that seven locations are found to show negative reddening values. Of these, two locations showed reddening values lower than -0.015 mag. In the statistical sense, this is a very small fraction of the total sample. The crosses in the figure denote the average reddening values as estimated by Udalski et al. (1999) and converted to $E(V-I)$. The error denotes the scatter in the reddening estimates of 4 subsections within each strip. These values are considerably larger than the values estimated in this study. This discrepancy is discussed in the following section.

\section{Results and discussion}

Udalski et al. (1999) presented average $E(B-V)$ reddening values for 84 sections, whereas $E(V-I)$ reddening values of 1123 sections are presented here, where both studies used the same data in the same region. The area used to estimate
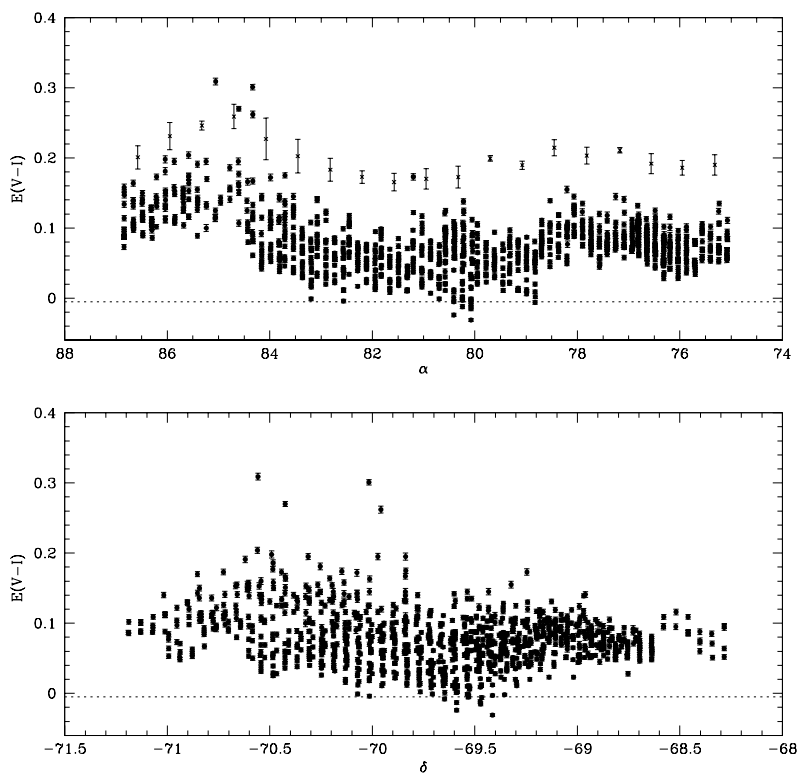

Fig. 5. $E(V-I)$ reddening values are shown as a function of $\alpha$ in the top panel and as a function of $\delta$ in the lower panel. The error in the estimation is also shown. The crosses denote the reddening estimation on Udalski et al. (1999), which is the average for each strip. The error denotes the scatter in the reddening estimated for four subsections in a strip.

average reddening in this study corresponds to $\sim 46 \mathrm{pc} \times 46 \mathrm{pc}$ (assuming $13.4 \mathrm{pc}$ for a distance modulus of $18.24 \mathrm{mag}$ ). 
On the other hand, the area averaged by Udalski et al. (1999) is $\sim 190 \mathrm{pc} \times 190 \mathrm{pc}$. Thus reddening values presented here have 16 times better spatial resolution. The values presented here are thus more suited for studies which require reddening estimates on smaller length scales.

The adopted value of $(V-I)_{0}=0.92$ needs some attention. This value has been adopted from Olsen \& Salyk (2002). They selected this value so as to produce a median reddening equal to that measured by Schlegel et al. (1998). Though none of the fields considered by Olsen \& Salyk (2002) fall within the bar, the assumption can be assumed to be valid considering the smooth distribution of reddening in the central region of the LMC. This may be a significant source of uncertainty in the final reddening values. Statistically, only $0.18 \%$ of regions showed negative reddening, which is a very negligible fraction. The fact that only two locations showed negative reddening implies that the assumed value of the mean colour of the red clump stars, which is the zero-point for reddening estimation, is not incorrect. The mean value of the intrinsic colour cannot be higher than the value assumed here, but at the same time, it does not rule out the possibility of a lower value. The mean colour of the red clump population in the solar neighbourhood was derived by Paczynski \& Stanek (1998), and was found to be $(V-I)_{\text {mean }}=1.01 \mathrm{mag}$. The mean colour for the bulge population of red clump stars was found to be $1.22 \mathrm{mag}$. This difference was interpreted as due to the bulge population having a broader range and a higher value of average metallicity than the local disk population. The fact that the mean colour of red clump stars in the LMC is found to be bluer (0.92 mag) could be explained as due to the red clump population in the LMC being comparatively metal poor than the local disk population. Girardi \& Salaris (2000) modeled the red clump population and estimated their mean $(V-I)$ and $M_{I}$ values as a function of age and metallicity. From their Fig. 1, it can be seen that for $Z=0.004$, the mean $(V-I)$ value is between 0.95 and $0.90 \mathrm{mag}$, for an age range of about $2-12 \mathrm{Gyr}$. This gives theoretical support to the mean value of $0.92 \mathrm{mag}$ assumed by Olsen \& Salyk (2002). The value of the mean colour was also found to increase with metallicity. If the mean colour of red clump stars was found to be different from the value used here, then the reddening of the locations presented here would shift by a value equal to the difference between the present zeropoint and the new value. The difference in reddening between two locations would still remain the same as estimated here.

The average value of $E(B-V)$ reddening as estimated by Udalski et al. (2000) was $0.143 \mathrm{mag}$, which would translate to $E(V-I)=1.4 E(B-V)=0.20 \mathrm{mag}$. This is very much higher than the value presented here. The average reddening presented in this study could be biased towards lower values due to the fact that regions with higher reddening are not considered. The average reddening values for each strip as estimated by Udalski et al. (1999) is shown in Fig. 5, where the $E(B-V)$ values are converted to $E(V-I)$ values using the relation $E(V-I)=1.4 E(B-V)$. It can be seen that the values estimated here are lower than those estimated by Udalski et al. (1999). There seems to be more or less a constant difference between the two values irrespective of location. The reason for this difference could be that the actual zero-point is

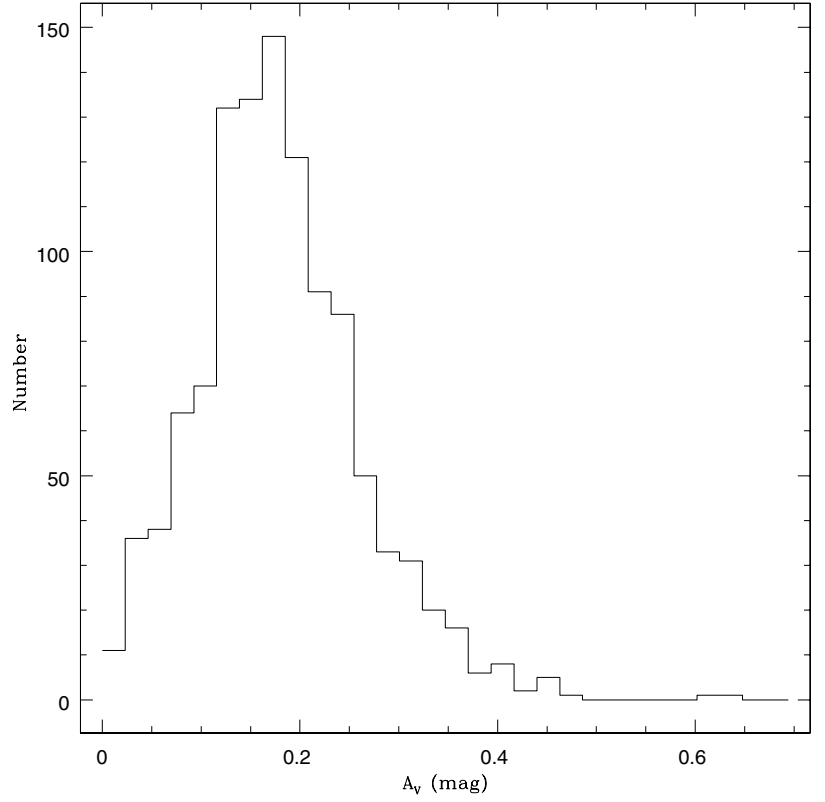

Fig. 6. A histogram of extinction, $A_{\mathrm{V}}$ mag. A single peaked distribution can be noticed.

lower than 0.92 mag by about $0.1 \mathrm{mag}$. Lowering of the zeropoint from 0.92 to $\sim 0.82$ would mean that the red clump stars have a metallicity around $Z=0.001$ (Girardi \& Salaris (2000). Harris et al. (1997) used around 2000 OB main-sequence stars to construct a map of the reddening in the region observed by Zaritsky et al. (1997). These regions are located to the north of the region used in this study. They find a mean reddening of $E(B-V)=0.20 \mathrm{mag}$. This would correspond to $E(V-I)=$ $0.28 \mathrm{mag}$ (assuming the relation $E(V-I)=1.4 E(B-V)$ ). Stanek et al. (1998) used red clump stars in two specific locations within the above region. The estimated values of reddening for these regions were $E(V-I)=0.20$ and $0.21 \mathrm{mag}$. It has been demonstrated by Zaritsky (1997) that the extinction property of the LMC varies both spatially and as a function of stellar population. Recently Zaritsky et al. (2004) presented an extinction map for two stellar populations in the LMC. These were obtained by fitting stellar atmospheric models to a set of cool and hot stars. They found that dust is highly localised near the younger, hotter stars, and in particular towards regions immediately east and northeast of the center of the LMC. Also, aside from the regions of higher extinction, no discernible global pattern was found. The reddening values estimated in the present study are slightly lower than those presented in the above reference. The stellar population studied here is predominantly located in the bar, which may be located in front of the dust and the gas clouds in the LMC. On the other hand, the stellar population analysed by Zaritsky et al. (2004) could belong to a younger population and hence be located in the disk, which may be mixed with gas and dust. Zaritsky et al. (2004) found that extinction towards older stars is bimodal such that it could be modeled as stars in front and behind a thin dust layer. For comparison, the histogram of the extinction in $V, A_{\mathrm{V}}$ is plotted in Fig. 6, where $A_{\mathrm{V}}$ is estimated as $A_{\mathrm{V}}=3.24 \times(E(V-I) / 1.4)$. It can be seen that the profile is similar to the profile presented by Zaritsky et al. (2004), but without the second peak. This again 
supports the fact that regions with higher reddening have been selectively omitted. This can therefore skew the value of average reddening towards lower values. The regions presented here thus represent locations which lie in front of the dust layer as mentioned by Zaritsky et al. (2004). They also mention that the Galactic foreground extinction is estimated to be minimal, of the order of $A_{\mathrm{V}} \sim 0.05 \mathrm{mag}$. The peak of the profile in Fig. 6 would shift marginally towards lower value when the Galactic foreground extinction is removed. Using the above relation, the $E(V-I)$ reddening due to the Galactic foreground can be estimated as $\sim 0.01 \mathrm{mag}$. Hence the major part of the reddening is due to the LMC itself.

A few locations near the center were found to show statistically significant negative reddening. This may indicate that the red clump population close to the center may be different from the rest of the bar region in terms of metallicity and/or age. If we consider near-zero reddening near these locations, then the mean $(V-I)$ required is 0.905 mag. From Girardi \& Salaris (2000, Fig. 1), the mean colour for a metallicity of $Z=0.001$ was found to be less than $0.85 \mathrm{mag}$. Therefore, for a population with similar age, the required increase in the metallicity would only be marginally more. If the metallicity were constant, then a red clump population with age younger than 1-2 Gyr is required to explain the observed colour. The above two ranges are quite possible, and hence the observed red clump population near the center could be either younger or slightly more metal rich than the rest of the population in the bar.

The reddening map as shown in Fig. 4 indicates that the central part of the bar region has very low reddening. This would mean that there is not much gas/dust present here. The reddening is more at the ends of the bar, with the eastern end having more reddening than the western end. Towards the west of the bar, the reddening increases initially, and then decreases to the values found near the center. Hence the presence of gas/dust are found only for a small stretch of the western bar. On the other hand, many locations in the eastern end of the bar show relatively high reddening. The reddening between $\alpha=84^{\circ}-85^{\circ}$ shows that a few regions have highest values of reddening. Further eastward of $85^{\circ}$, the reddening is found to be slightly higher, but with similar dispersion as seen in the rest of the bar. This indicates that only the net reddening is higher, whereas the dispersion, which corresponds to differential reddening, is not higher. This can be clearly seen from the middle panel of Fig. 3, where the value of $\sigma$ is not found to be higher for the eastern end, although a few points show higher values. The top panel of Fig. 5 also indicates that a higher reddening is found towards the east, similar to the result of Zaritsky et al. (2004). We try to find the possible reason for this increased reddening. The H I density map presented in Fig. 4 of Kim et al. (1998) shows a very high density of $\mathrm{H} \mathrm{I}$ at $\alpha \sim 85^{\circ}$ and $\delta$ between $69^{\circ}-71^{\circ}$. This dense gas should have caused very high reddening in these regions. The reddening values obtained here should then be very high, rather than the estimated small increase. Thus the higher reddening observed for the east, when compared to the west, can be explained as due to the presence of an increased amount of gas and dust in the eastern region. On the other hand, the reddening observed is not too high to account for the very large amount of gas as observed by
Kim et al. (1998). Thus we find that only a small fraction of the $\mathrm{H}$ I gas seems to be located in front of the bar, giving rise to increased reddening. Most importantly, the high column density of $\mathrm{H}$ I clouds seen in this direction is most likely to be located behind the bar region.

The main results are summarised as follows:

The $E(V-I)$ reddening values of 1123 locations in the bar region of the LMC are presented here. These are estimated using the red clump stars identified from the OGLE II catalogue. These estimations can be considered to be homogeneous with minimum systematic errors.

The reddening values were found to have values between 0.1 and $0.3 \mathrm{mag}$, with an average value of $0.08 \pm 0.04 \mathrm{mag}$, indicating a more or less uniform reddening throughout the bar region. The average presented here is likely to be skewed towards lower values of reddening, due to selective omission of highly reddened regions.

The eastern end of the bar is found to have slightly increased reddening with respect to the rest of the bar. This indicates that there is some amount of gas/dust located in front of the bar. On the other hand, this increased reddening is not enough to account for the high HI column density observed. Therefore, most of the $\mathrm{H}$ I clouds seen in the $\mathrm{H}$ I maps are likely to be located behind the bar.

Acknowledgements. I thank Prof. N. K. Rao for suggesting to publish the results presented here. I also thank the referee improving the discussion in the paper.

\section{References}

Alcock, C., Allsman, R. A., Alves, D. R., et al. 2000, AJ, 119, 2194

Cole, A. A. 1998, ApJ, 500, 137

de Vaucouleurs, G., \& Freeman, K. C. 1973, Vistas Astron., 14, 163

Girardi, L., \& Salaris, M. 2001, MNRAS, 323, 109

Harris, J., Zaritsky, D., \& Thompson, I. 1997, AJ, 114, 1933

Kim, S., Staveley-Smith, L., Dopita, M. A., et al. 1998, ApJ, 503, 674

Olsen, K. A. G., \& Salyk, C. 2002, AJ, 124, 2045

Paczynski, B., \& Stanek, K. Z. 1998, ApJ, 494, L219

Popowski, P., Cook, K. H., \& Becker, A. C. 2003, AJ, 126, 2910

Schlegel, D. J., Finkbeiner, D. P., \& Davis, M. 1998, ApJ, 500, 525

Stanek, K. Z. 1996, ApJ, 460, 37

Stanek, K. Z., Zaritsky, D., \& Harris, J. 1998, ApJ, 500, 141

Subramaniam, A., \& Anupama, G. C. 2002, A\&A, 390, 449

Subramaniam, A. 2004, ApJ, 604, L41

Subramaniam, A. 2003, ApJ, 598, L19

Sumi, T. 2004, MNRAS, 349, 193

Udalski, A. 2000, Acta Astron., 50, 279

Udalski, A., Szymanski, M., Kubiak, M., et al. 2000, Acta Astron., 50, 307 (OGLE II data)

Udalski, A., Soszynski, I., Szymanski, M., et al. 1999, Acta Astron., 49, 223

Udalski, A., Szymanski, M., Kubiak, M., et al. 1998, Acta Astron., 48, 1

van der Marel, R. P., \& Cioni, M.-R. 2001, AJ, 122, 1807

Wozniak, P. R., \& Stanek, K. Z. 1996, ApJ, 464, 233

Zaritsky, D., Harris, J.,\& Thompson, I. 1997, AJ, 114, 1003

Zaritsky, D. 1999, AJ, 195, 6801

Zaritsky, D., Harris, J., Thompson, I. B., \& Grebel, E. K. 2004, AJ, 128,1606 